\title{
Clinical analysis of 16 cases of pulmonary cryptococcosis in patients with normal immune function
}

\author{
Jie Zhang ${ }^{1 \#}$, Duchao Zhang ${ }^{1 \#}$, Xinying Xue ${ }^{1}$, Lei Yang ${ }^{2}$, Liang Chen ${ }^{3}$, Lei Pan ${ }^{1}$ \\ ${ }^{1}$ Department of Pulmonary and Critical Care Medicine, ${ }^{2}$ Department of Radiology, ${ }^{3}$ Department of Laboratory Medicine, Beijing Shijitan Hospital, \\ Capital Medical University, Beijing 100038, China \\ Contributions: (I) Conception and design: J Zhang, D Zhang, L Pan; (II) Administrative support: J Zhang, L Pan; (III) Provision of study materials or \\ patients: J Zhang, D Zhang, X Xue, L Yang, L Chen; (IV) Collection and assembly of data: J Zhang, D Zhang; (V) Data analysis and interpretation: J \\ Zhang, D Zhang, L Pan; (VI) Manuscript writing: All authors; (VII) Final approval of manuscript: All authors. \\ "These authors contributed equally to this work as co-lead authors. \\ Correspondence to: Lei Pan. Department of Pulmonary and Critical Care Medicine, Beijing Shijitan Hospital, Capital Medical University, Beijing \\ 100038, China. Email: leipan61@aliyun.com.
}

Background: Pulmonary cryptococcosis (PC) is an invasive pulmonary fungal disease caused by Cryptococcus neoformans or Cryptococcus gattii. Cryptococcus is the opportunistic pathogen. Immune damage and chronic consumptive diseases are the high risk. The prevalence of cryptococcus infection is $5 \%$ to $10 \%$ in individuals with weakened immune function. In recent years, with the application of glucocorticoids, antitumor drugs, and broad-spectrum antibiotics, the prevalence of cryptococcal infection has increased yearly. Cryptococcus infection can also occur in normal people without underlying diseases. One in every 100,000 individuals with normal immune function is also affected, but these cases are often misdiagnosed due to inadequate understanding and low awareness of PC in individuals with normal immune function. In this study, we investigated the clinical characteristics of PC in patients with normal immune function to improve the diagnosis and treatment of these cases.

Methods: We retrospectively analyzed the clinical, imaging and pathological data of 16 patients with pulmonary cryptococcosis treated at Beijing Shijitan Hospital between 2010 and 2017.

Results: Of the 16 patients with disseminated cryptococcosis, 6 were men, and 10 were women; their ages were 35 to 76 years (median: 53 years). Initial symptoms were cough, sputum, chest pain, and fever. Thirteen patients had a history of chronic disease or tumor, including diabetes and bronchial asthma $(\mathrm{n}=2)$, simple hypertension $(n=2)$, chronic bronchitis $(n=2)$, diabetes and hypertension $(n=5)$, previous tuberculosis $(n=1)$, and previous lung cancer surgery $(n=1)$. Chest computed tomography $(C T)$ in these 16 patients showed single or multiple nodules and masses $(n=14)$, lobar consolidation $(n=1)$, and diffuse mixed lesions $(n=1)$. Lung biopsy showed granulomatous lesions in these 16 patients. Cryptococcus neoformans was identified with Grocott's methenamine silver and Grocott's methenamine silver (GMS) stains. Fourteen patients received fluconazole alone; two patients with intracranial infection received fluconazole combined with amphotericin B. Five patients improved significantly, and nine patients improved with significant lesion absorption.

Conclusions: The clinical manifestations of PC are nonspecific in patients with normal immune function. Lung biopsy and cryptococcus antigen tests of cerebrospinal fluid are valuable diagnostic aids. Fluconazole is an effective treatment for disseminated cryptococcal infection.

Keywords: Pulmonary cryptococcosis (PC); immune function; clinical analysis

Submitted Mar 03, 2020. Accepted for publication May 06, 2020.

doi: 10.21037/apm-20-897

View this article at: http://dx.doi.org/10.21037/apm-20-897 


\section{Introduction}

Cryptococcosis is a widespread opportunistic fungal infection caused by Cryptococcus neoformans or Cryptococcus gattii, is common in immunocompromised individuals. The most common susceptibility factor is human immunodeficiency virus (HIV) infection, and those with acquired immunodeficiency syndrome (AIDS) are especially susceptible. In recent years, with the application of glucocorticoids, immunosuppressants, and antitumor drugs, the prevalence of cryptococcal infection has increased yearly. The prevalence of cryptococcus infection is $5 \%$ to $10 \%$ in individuals with weakened immune function. Cryptococcus infection can also occur in normal people without underlying diseases. One in every 100,000 individuals with normal immune function is also affected. Some reports have shown that cryptococcosis, especially pulmonary cryptococcosis, occurs more frequently in immunocompetent patients than in immunocompromised ones $(1,2)$.

Cryptococcus usually infects the lungs, central nervous system, and skin. Pulmonary cryptococcosis (PC) is often misdiagnosed as lung cancer, tuberculosis, or nonspecific lung inflammation due to the lack of specific clinical and imaging findings (3). In the population with normal immune function, the misdiagnosis rate increased significantly. PC is confirmed mainly by pathological examination $(3,4)$ of surgical specimens, percutaneous lung biopsy samples, or bronchoscopic biopsy samples. The treatment of PC includes surgery and medical treatment (5-7). Surgical treatment has the advantages of radical local resection and a short treatment period. Antifungal drugs include amphotericin B, 5-fluorocytosine (5-FC), clotrimazole and fluconazole.

Because of the nonspecific clinical and imaging features of pulmonary cryptococcosis, especially in the immunocompetent population, the low vigilance will lead to the untimely diagnosis and treatment. In this study, we analyzed the epidemiological, clinical, radiological characteristics, as well as treatments of 16 PC patients with normal immune function who were treated at our hospital to deepen the understanding of PC in individuals with normal immune function. We present the following article in accordance with the STROBE reporting checklist (available at http://dx.doi.org/10.21037/apm-20-897).

\section{Methods}

A total of 16 PC patients (including inpatients and outpatients) treated in Beijing Shijitan Hospital, Capital
Medical University, between January 2010 and December 2017 were included in this study. All cases were confirmed with biopsy (percutaneous lung biopsy: $n=14$, bronchoscopic biopsy: $\mathrm{n}=2$ ). Clinical data, including sex, age at onset, underlying diseases, respiratory symptoms, imaging results, treatment, and follow-up data were analyzed. For the pathological examination, all 16 specimens were fixed with $4 \%$ neutral formaldehyde, embedded in paraffin, and stained with hematoxylin and eosin (HE). Immunohistochemistry was performed with periodic acid-Schiff (PAS) and Grocott's methenamine silver (GMS) stains, followed by examination under a light microscope.

\section{Results}

\section{Clinical manifestations}

Of the 16 PC patients, 6 were men, and 10 were women; they ranged in age from 35 to 76 years (median: 53 years). Initial symptoms were cough $(16,100 \%)$, sputum (13, $81.3 \%)$, chest pain $(6,37.5 \%)$, fever $(4,25.0 \%)$, and headache $(2,12.5 \%)$. Thirteen patients had a history of chronic disease or tumor, including diabetes and bronchial asthma $(n=2)$, chronic bronchitis $(n=2)$, simple hypertension $(\mathrm{n}=2)$, diabetes and hypertension $(\mathrm{n}=5)$, previous tuberculosis $(\mathrm{n}=1)$, and previous lung cancer surgery $(\mathrm{n}=1)$. None of the patients had prior use of steroids or immunosuppressants. Two patients had a history of close contact with birds. Lymphocyte subpopulations were normal, and HIV antibodies (HIV-Ab) were negative in all 16 patients (Table 1).

\section{Imaging findings}

Chest CT showed abnormal opacities in the lungs in all 16 patients (Tables 2 and 3; Figure 1), including single or multiple nodules and masses $(n=14)$, lobar consolidation $(\mathrm{n}=1)$, and diffuse mixed lesions $(\mathrm{n}=1)$. Most lesions were in the lower lobes; these included solitary lesions $(n=3)$, unilateral multiple lesions $(n=8)$, and bilateral lesions $(n=5)$. In terms of morphology, (I) chest CT showed nodules and masses in 14 cases, including solitary lesions $(\mathrm{n}=2)$ and multiple lesions ( $n=12)$. Most of these lesions were in the outer one-third of the lung fields and ranged in size from 0.4 to $6.0 \mathrm{~cm}$. The edges were smooth in 9 cases and irregular in 5 cases (including burr sign in 2 cases). The density was even in 11 cases and uneven in 3 cases. In addition, CT showed cavities in 4 cases and air bronchogram signs in 
Table 1 Underlying diseases and clinical manifestations of PC patients

\begin{tabular}{lc}
\hline Item & $\mathrm{n}(\%)$ \\
\hline Underlying disease & $13(81.25)$ \\
Chronic bronchitis & $2(12.5)$ \\
Bronchial asthma & $2(12.5)$ \\
Previous tuberculosis & $1(6.25)$ \\
Postsurgery lung cancer & $1(6.25)$ \\
Type 2 diabetes & $7(43.75)$ \\
Hypertension & $5(31.25)$ \\
Clinical manifestation & \\
Cough & $16(100.0)$ \\
Sputum & $13(81.25)$ \\
Chest pain & $6(37.5)$ \\
Fever & $4(25.0)$ \\
Headache & $2(12.5)$ \\
\hline
\end{tabular}

PC, pulmonary cryptococcosis.

Table 2 Four types of CT findings in PC patients

\begin{tabular}{|c|c|c|}
\hline PC type & n (\%) & CT findings \\
\hline $\begin{array}{l}\text { Solitary } \\
\text { nodule or } \\
\text { mass }\end{array}$ & $2(12.5)$ & $\begin{array}{l}\text { The lesion was in the outer one-third of } \\
\text { the lung field, and the base was next } \\
\text { to the pleura, with a cavity (thick and } \\
\text { smooth wall) and air bronchogram sign }\end{array}$ \\
\hline $\begin{array}{l}\text { Multiple } \\
\text { nodules or } \\
\text { masses }\end{array}$ & $12(75.0)$ & $\begin{array}{l}\text { Most lesions were in the lower lobes } \\
\text { and outer one-third of the lung field. } \\
\text { Some lesions had irregular edges, } \\
\text { burrs, pleural stretching, and cavities } \\
\text { with a thick wall }\end{array}$ \\
\hline Consolidation & $1(6.25)$ & $\begin{array}{l}\text { Wedge-shaped consolidation } \\
\text { opacities with uneven density and air } \\
\text { bronchogram sign }\end{array}$ \\
\hline $\begin{array}{l}\text { Diffuse mixed } \\
\text { lesion }\end{array}$ & $1(6.25)$ & $\begin{array}{l}\text { Mixed, clustered, patchy, nodular, mass, } \\
\text { and consolidation opacities, mostly in } \\
\text { the outer one-third of the lung field and } \\
\text { lower lobes, with air bronchogram sign } \\
\text { in consolidation lesions }\end{array}$ \\
\hline
\end{tabular}

PC, pulmonary cryptococcosis.

5 cases. (II) CT showed a single lobar consolidation in one patient, with air bronchogram sign and uneven density. (III) The case with diffuse mixed lesions presented bilateral irregular, patchy, nodular, and mass opacities with pleural
Table 3 CT imaging findings of nodular or mass opacities

\begin{tabular}{ll}
\hline Item & $\mathrm{n}$ \\
\hline Location & 4 \\
Upper middle lobe & 9 \\
Lower lobe & 1 \\
Entire lung(s) & 2 \\
Unilateral & 12 \\
Bilateral & \\
Morphology & 6 \\
Mass & 8 \\
Nodule & 3 \\
Lobe & 2 \\
Burr & 4 \\
Cavity & 2 \\
Pleural stretching & 5 \\
Air bronchogram sign & \\
\hline
\end{tabular}

effusion, predominantly in the lower lobes.

\section{Pathology}

All 16 patients underwent biopsy, including percutaneous biopsy (n=14) and bronchoscopic biopsy $(\mathrm{n}=2)$ (Figure 2). Two cases were confirmed with rapid cryptococcus antigen testing of cerebrospinal fluid. In addition to the infiltration of macrophages, lymphocytes, and plasma cells, granulomatous lesions (as per pathological examinations of paraffin sections) showed diffuse infiltration of multinucleated giant cells, with round vacuole-like cryptococcus spores in the cytoplasm. The spores varied in size and were clustered in the cytoplasm of multinucleated giant cells. HE staining showed that the bacterium was slightly basophilic and difficult to identify. PAS staining (red) and GMS staining (dark brown) showed cryptococcus spores with a thick wall. Two patients tested positive on rapid cryptococcus antigen tests of cerebrospinal fluid collected via lumbar puncture.

\section{Outcome and follow-up}

Fourteen patients received fluconazole alone (200 to $400 \mathrm{mg} /$ day, 6 to 12 months); two patients with intracranial 

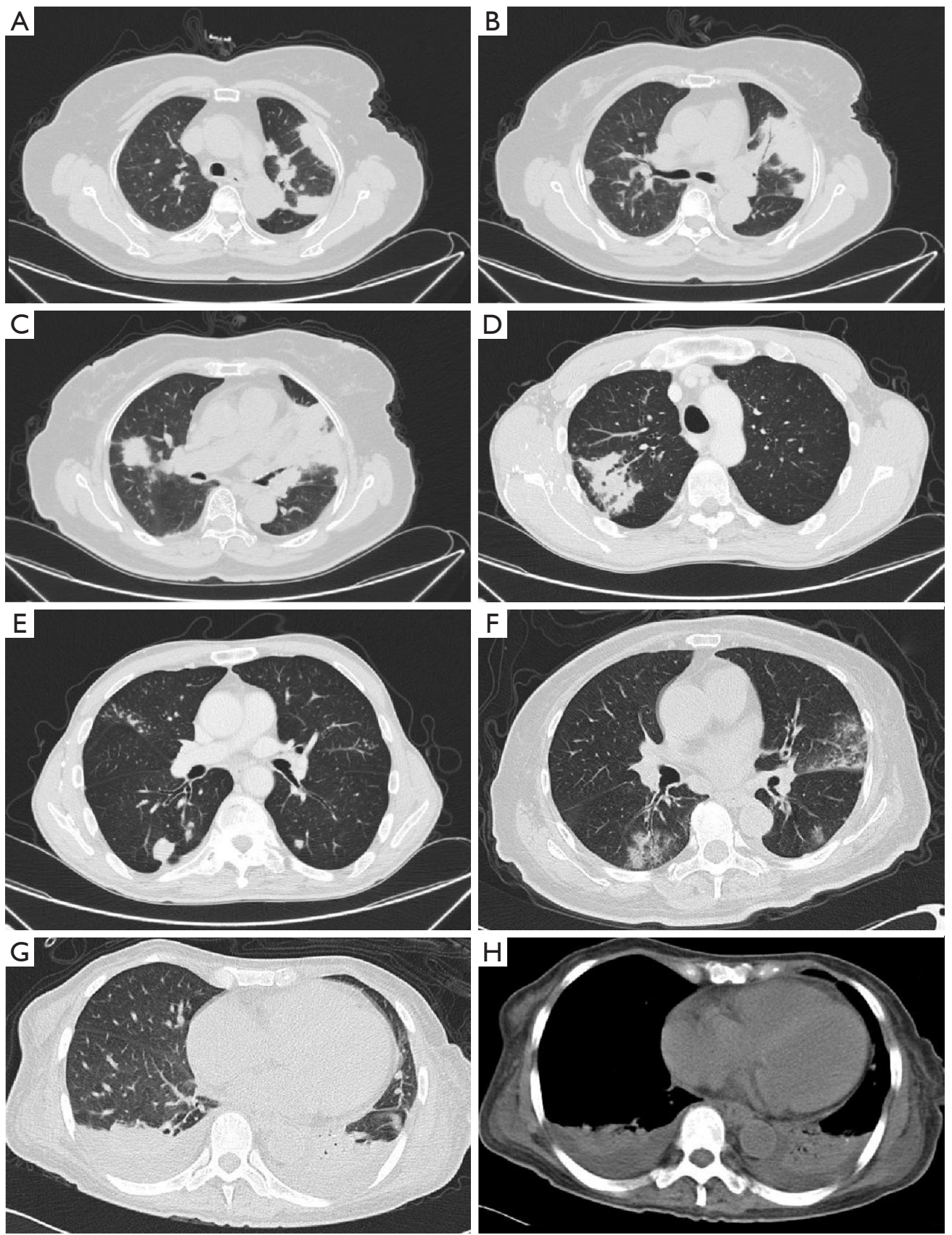

Figure 1 CT scans of pulmonary cryptococcosis. (A) Multiple masses in the left upper lobe. The base was next to the pleura, with burrs. (B) Bilateral multiple nodules and consolidation opacities with air bronchogram sign. (C) Wedge-shaped consolidation opacity in the left lung and mass opacity in the right lung, with lobes and burrs. (D) Mass opacity in the right lung with a cavity (smooth wall). (E) Subpleural nodule with burrs and pleural stretching. (F) Bilateral multiple ground-glass opacities. (G and H) Bilateral consolidation opacities with pleural effusion. 

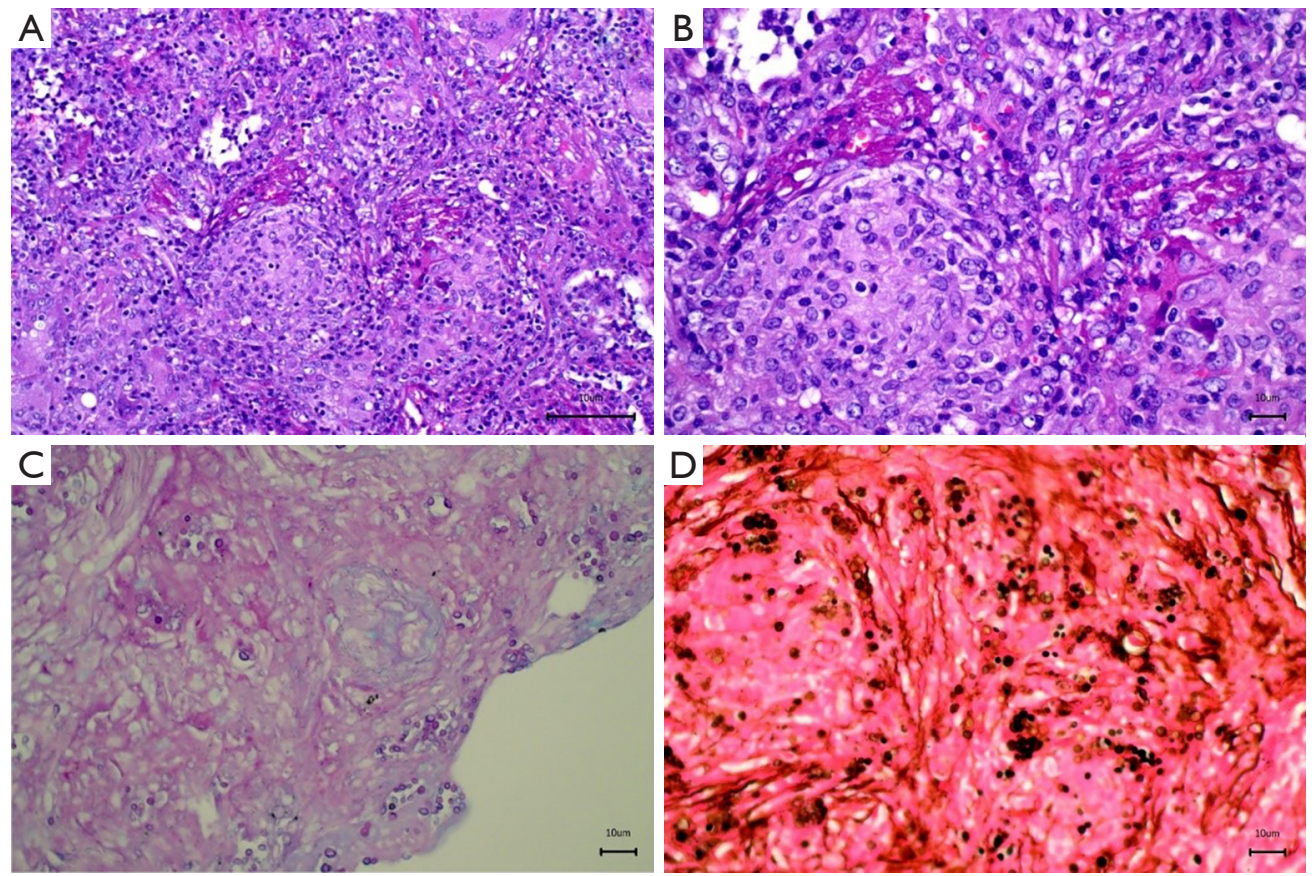

Figure 2 Histopathology of pulmonary cryptococcosis. (A and B) HE staining showed granulomatous lesions with round vacuole-like cryptococcus spores, which was slightly basophilic and difficult to identify. (C) PAS staining (red) and (D) GMS staining (dark brown) showed cryptococcus spores with a thick wall.

infection received induction therapy with fluconazole (200 mg/day) and amphotericin B (0.7 to $1 \mathrm{mg} / \mathrm{kg} /$ day $)$ for 4 weeks, consolidation therapy with fluconazole (400 $\mathrm{mg} /$ day) for 8 weeks, and maintenance therapy with fluconazole $(200 \mathrm{mg} /$ day $)$. Five patients improved significantly, and nine patients improved with significant lesion absorption (Figure 3), with no recurrence during the 1- to 2-year follow-up.

\section{Discussion}

Cryptococcosis is an opportunistic infection caused mainly by Cryptococcus neoformans or Cryptococcus gattii. Cryptococcus is a yeast that is widely distributed as a parasitic fungus in nature and is present in pigeon manure and soil. Available data show that susceptible populations include patients with chronic diseases or long-term use of immunosuppressants, cytotoxic drugs, glucocorticoids, or antibiotics. In recent years, the incidence of $\mathrm{PC}$ in patients with normal immune function (no apparent underlying disease or predisposing factors) has increased; this population now accounts for approximately one-third of all cases $(3,8)$. The host's defense against cryptococcus infection mainly depends on the immune system mediated by monocyte macrophages and $\mathrm{T}$ lymphocytes. In immunodeficient patients with PC, studies have shown that deficiency in CD4+ T cells or the impaired anticryptococcal activity of monocytes will lead to high prevalence of cryptococcosis $(9,10)$. In immunocompetent patients with $\mathrm{PC}$, studies suggest that Cryptococcus possibly resides within the host and may be inhibiting their protective immune response (11-13). In this study, two patients had a history of contact with birds. Lymphocyte subpopulations were normal in all 16 patients. Three patients (3/16) had no history of underlying disease or predisposing factors, indicating that PC may occur in individuals with normal immune function.

The initial clinical manifestations of PC are usually respiratory symptoms, including cough, sputum, fever, hemoptysis, chest pain, and headache, which are similar to those of other respiratory diseases such as lung cancer, pneumonia, and tuberculosis (14-16). As a result, PC is often misdiagnosed, and some asymptomatic cases are detected on chest CT performed during routine checkups. In this study, initial symptoms were nonspecific and included cough $(16,100.0 \%)$, sputum $(13,81.2 \%)$, chest pain $(6,37.5 \%)$, and fever $(4,25.0 \%)$, which is consistent 

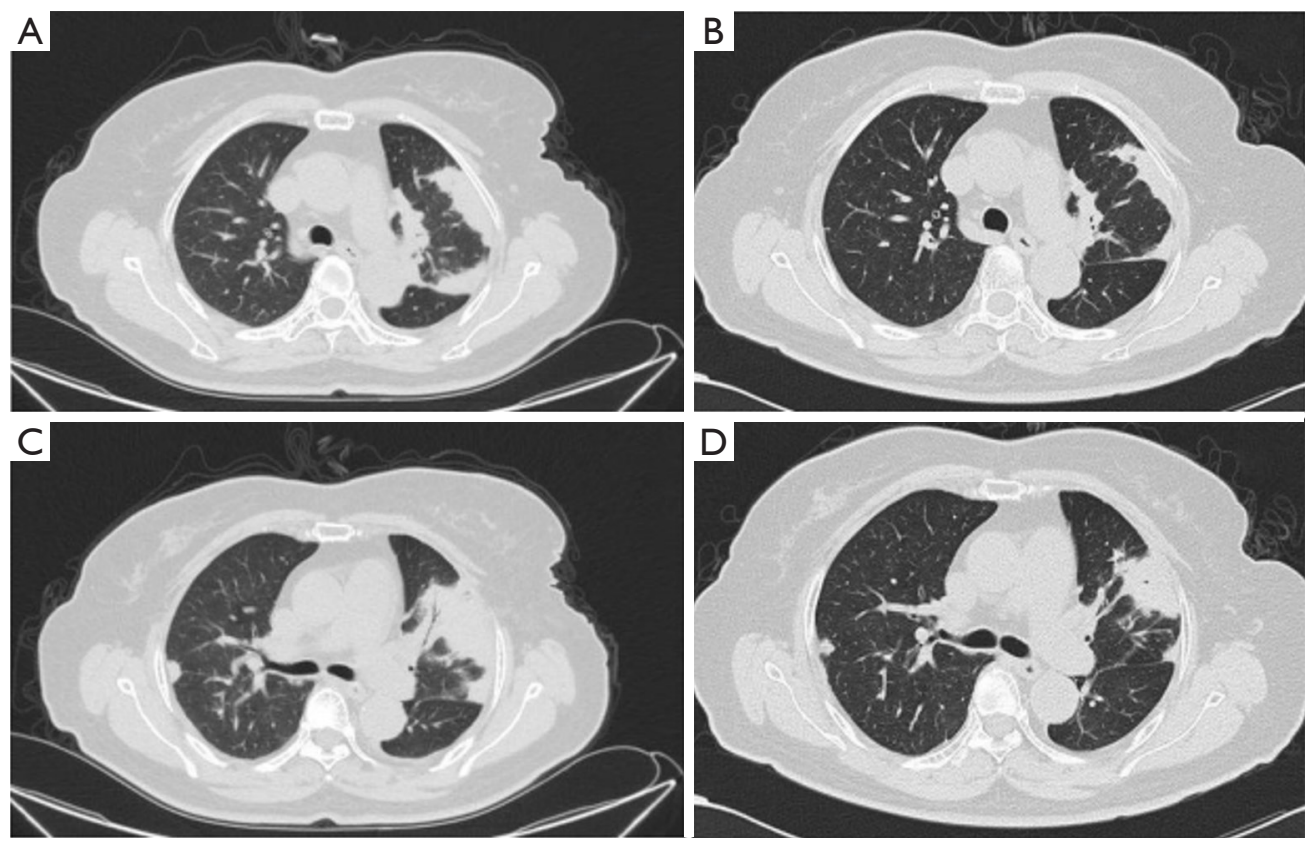

Figure 3 CT scans before and after treatment of PC. (A and C) Before treatment; (B and D) after 3 months of treatment. PC, pulmonary cryptococcosis.

with literature reports. Cryptococcus has a high affinity for the central nervous system (6). In this study, two patients $(12.5 \%)$ had meningitis, indicating that clinicians should pay attention to this possibility, especially in patients with headache.

Chest CT findings of PC vary from patient to patient (14-17). The most common findings are solitary or multiple nodules or mass opacities ranging in size from 0.5 to $10 \mathrm{~cm}$. The clinical manifestations vary, and infection is often misdiagnosed as lung cancer due to features such as a lobular shape, burrs, and cavities. In this study, CT showed nodules or mass opacities in 14 cases $(87.5 \%)$. Most lesions were in the lower lobes (rarely in middle lobes) and in the outer one-third of the lung field, and the base was next to the pleura in most cases. Previous reports show that in PC cases, masses or nodules may have central necrosis or liquefaction, forming cavities with a thick wall after the release of necrotic tissue $(14,17)$. In this study, four patients had cavities with a smooth and thick (up to $4 \mathrm{~cm}$ ) wall. Another important sign is the air bronchogram sign, which was found in 5 cases in this study. This sign occurs as a result of granulomatous inflammation with an intact bronchial structure. Compared with lung cancer, nodules or mass lesions with PC are more common in the outer onethird of the lung field, distributed in clusters, with uniform density, low tension, rare deep lobulation, and more air bronchogram sign. Pulmonary consolidation opacity is characterized by wedge-shaped subpleural consolidation opacity with uneven density, and the base is located next to the pleura. Diffuse mixed lesions vary and may present as mixed, patchy nodules or masses and consolidation opacities. Most lesions are in the outer one-third of the lung field. In this study, one patient had mixed lesions with air bronchogram sign in a consolidation opacity and bilateral pleural effusion, features that must be differentiated from pneumonia and tuberculosis.

Due to its nonspecific clinical manifestations and varying chest CT findings, PC is often misdiagnosed as lung cancer, bacterial pneumonia, or tuberculosis. PC is confirmed mainly by pathological examination $(3,4)$ of surgical specimens, percutaneous lung biopsy samples, or bronchoscopic biopsy samples; respiratory smears or cultures; cerebrospinal fluid smears or cultures; and pus smears or cultures. This study showed that most cases were confirmed by percutaneous lung biopsy and bronchoscopic biopsy. In addition, for patients with central nervous system symptoms such as headache, nausea, and vomiting, it is important to perform cerebrospinal fluid smears and cultures to exclude central nervous system infection.

$\mathrm{PC}$ is treated with surgical removal and medical 
treatment (5-7). Surgical treatment has the advantages of radical local resection and a short treatment period. Antifungal drugs include amphotericin B, 5-fluorocytosine (5-FC), clotrimazole and fluconazole. Some recent studies have shown that fluconazole is effective, has relatively few adverse reactions, and may be used as a routine treatment for PC. It is important that patients receive a full course of treatment, usually 6 to 12 months. In this study, all 16 patients, including two with intracranial infection, received full-course treatment. Five patients improved significantly, and nine patients improved with significant lesion absorption, with no recurrence during the 1- to 2-year follow-up.

In summary, the clinical manifestations of PC are nonspecific in patients with normal immune function. The imaging findings are usually nodules or mass in the outer one-third of the lung field, which are distributed in clusters, with uniform density and air bronchogram sign. Lung biopsy and cryptococcus antigen tests of cerebrospinal fluid are valuable diagnostic aids. Fluconazole is effective for the treatment of PC.

\section{Limitations}

There are a number of limitations that needs to be addressed in this study. Although the sample is representative, the samples were mall, we will expand the sample size and conduct multicenter research. This study is a retrospective study. In the future, we will further explore the mechanism of cryptococcus infection in people with normal immune function.

\section{Acknowledgments}

Funding: Promotion of Medical Service and Guarantee Capacity in 2019 (Capacity Building of Medical and Health Institutions) (z155080000004).

\section{Footnote}

Reporting Checklist: The authors have completed the STROBE Reporting Checklist. Available at http://dx.doi. org/10.21037/apm-20-897

Data Sharing Statement: Available at http://dx.doi. org/10.21037/apm-20-897

Conflicts of Interest: All authors have completed the ICMJE uniform disclosure form (available at http://dx.doi. org/10.21037/apm-20-897). The authors have no conflicts of interest to declare.

Etbical Statement: The authors are accountable for all aspects of the work in ensuring that questions related to the accuracy or integrity of any part of the work are appropriately investigated and resolved. This is a retrospective analysis of clinical case data and involves no interventions. Thus, this study poses no physiological risk to patients. Every effort is taken to protect the confidentiality of information from patients. A waiver of informed consent has been granted.

Open Access Statement: This is an Open Access article distributed in accordance with the Creative Commons Attribution-NonCommercial-NoDerivs 4.0 International License (CC BY-NC-ND 4.0), which permits the noncommercial replication and distribution of the article with the strict proviso that no changes or edits are made and the original work is properly cited (including links to both the formal publication through the relevant DOI and the license). See: https://creativecommons.org/licenses/by-nc-nd/4.0/.

\section{References}

1. Liu K, Ding H, Xu B, et al. Clinical analysis of nonAIDS patients pathologically diagnosed with pulmonary cryptococcosis. J Thorac Dis 2016;8:2813-21.

2. Yang B, Lee H, Lee T, et al. The use of surgery in a real-world clinic to diagnose and treat pulmonary cryptococcosis in immunocompetent patients. J Thorac Dis 2019;11:1251-60.

3. Zhou Y, Lin PC, Ye JR, et al. The performance of serum cryptococcal capsular polysaccharide antigen test, histopathology and culture of the lung tissue for diagnosis of pulmonary cryptococcosis in patients without HIV infection. Infect Drug Resist 2018;11:2483-90.

4. Lu J, Palavecino EL, Salih ZT. Pulmonary cryptococcosis diagnosed on a lung fine-needle aspiration. Diagn Cytopathol 2018;46:160-1.

5. Santos-Gandelman J, Rodrigues ML, Machado Silva A. Future perspectives for cryptococcosis treatment. Expert Opin Ther Pat 2018;28:625-34.

6. Ribeiro NQ, Santos APN, Emidio ECP, et al. Pioglitazone as an adjuvant of amphotericin $\mathrm{B}$ for the treatment of cryptococcosis. Int J Antimicrob Agents 2019;54:301-8.

7. Henao-Martínez AF, Chastain DB, FrancoParedes C. Treatment of cryptococcosis in non-HIV 
immunocompromised patients. Curr Opin Infect Dis 2018;31:278-85.

8. Kanjanapradit K, Kosjerina Z, Tanomkiat W, et al. Pulmonary Cryptococcosis Presenting With Lung Mass: Report of 7 Cases and Review of Literature. Clin Med Insights Pathol 2017;10:1179555717722962.

9. Olszewski MA, Zhang Y, Huffnagle GB. Mechanisms of cryptococcal virulence and persistence. Future Microbiol 2010;5:1269-88.

10. Koguchi Y, Kawakami K. Cryptococcal infection and Th1Th2 cytokine balance. Int Rev Immunol 2002;21:423-38.

11. Kobayashi M, Murata K, Hiroshi HO, et al. Cryptococcosis: long-lasting presence of fungi after successful treatment. Acta Derm Venereol 2004;84:320-21.

12. Spitzer ED, Spitzer SG, Freundlich LF, et al. Persistence of initial infection in recurrent Cryptococcus neoformans meningitis. Lancet 1993;341:595-6.

13. Wang J, Zeng Y, Luo W, et al. The Role of Cryptococcus in the Immune System of Pulmonary Cryptococcosis Patients. PLoS One 2015; 10:e0144427.

14. Deng H, Zhang J, Li J, et al. Clinical features and radiological characteristics of pulmonary cryptococcosis. J Int Med Res 2018;46:2687-95.

15. Taniwaki $M$, Yamasaki $M$, Ishikawa $N$, et al. Pulmonary cryptococcosis mimicking lung cancer. Lancet Infect Dis 2019;19:1033.

16. Huang Y, Sui X, Song L, et al. Imaging Findings of Pulmonary Cryptococcosis. Zhongguo Yi Xue Ke Xue Yuan Xue Bao 2019;41:832-6.

17. Li A, Li Q, Guo C, et al. Asymptomatic Meningitis and Lung Cavity in a Case of Cryptococcosis. Am J Case Rep 2017;18:1140-4.

Cite this article as: Zhang J, Zhang D, Xue X, Yang L, Chen L, Pan L. Clinical analysis of 16 cases of pulmonary cryptococcosis in patients with normal immune function. Ann Palliat Med 2020;9(3):1117-1124. doi: 10.21037/apm-20-897 